Commun.Fac.Sci.Univ.Ank.Series A 1

Volume 61, Number 2, Pages 1-8 (2012)

ISSN $1303-5991$

\title{
SOME PROPERTIES OF RICKART MODULES
}

\author{
B. ÜNGÖR, G. KAFKAS, S. HALICIOĞLU AND A. HARMANCI
}

\begin{abstract}
Let $R$ be an arbitrary ring with identity and $M$ a right $R$-module with $S=\operatorname{End}_{R}(M)$. Following [8], the module $M$ is called Rickart if for any $f \in S, r_{M}(f)=e M$ for some $e^{2}=e \in S$, equivalently, $\operatorname{Ker} f$ is a direct summand of $M$. In this paper, we continue to investigate properties of Rickart modules. For a Rickart module $M$, we prove that $M$ is $S$-rigid (resp., $S$ reduced, $S$-symmetric, $S$-semicommutative, $S$-Armendariz) if and only if its endomorphism ring $S$ is rigid (resp., reduced, symmetric, semicommutative, Armendariz). We also prove that if $M[x]$ is a Rickart module with respect to $S[x]$, then $M$ is Rickart, the converse holds if $M$ is $S$-Armendariz. Among others it is also shown that $M$ is a Rickart module if and only if every right $R$-module is $M$-principally projective.
\end{abstract}

\section{INTRODUCTION}

Throughout this paper $R$ denotes an associative ring with identity and modules will be unitary right $R$-modules. For a module $M, S=\operatorname{End}_{R}(M)$ denotes the ring of right $R$-module endomorphisms of $M$. Then $M$ is a left $S$-module, right $R$-module and $(S, R)$-bimodule. In this work, for any rings $S$ and $R$ and any $(S, R)$-bimodule $M, r_{R}($.$) and l_{M}($.$) denote the right annihilator of a subset of M$ in $R$ and the left annihilator of a subset of $R$ in $M$, respectively. Similarly, $l_{S}($. and $r_{M}($.$) will be the left annihilator of a subset of M$ in $S$ and the right annihilator of a subset of $S$ in $M$, respectively. A ring $R$ is reduced if it has no nonzero nilpotent elements. A ring $R$ is called semicommutative if for any $a, b \in R, a b=0$ implies $a R b=0$. The module $M$ is called $S$-semicommutative [2], if for any $f \in S$ and $m \in M, f m=0$ implies $f S m=0$. Baer rings [3] are introduced as rings in which the right (left) annihilator of every nonempty subset is generated by an idempotent. According to Rizvi and Roman, an $R$-module $M$ is called Baer [7]

Received by the editors March 08, 2012, Accepted:Oct. 08, 2012.

2010 Mathematics Subject Classification. : 13C99, 16D80, 16U80.

Key words and phrases. Rickart modules, symmetric modules, reduced modules, rigid modules, semicommutative modules, Armendariz modules. 
if for any $R$-submodule $N$ of $M, l_{S}(N)=S e$ with $e^{2}=e \in S$. Also, they defined Rickart modules in [8]. Recently Rickart modules are studied extensively by different authors (see [1] and [5]).

\section{Rickart Modules}

Let $M$ be an $R$-module with $S=\operatorname{End}_{R}(M)$. The module $M$ is called Rickart if for any $f \in S, r_{M}(f)=e M$ for some $e^{2}=e \in S$, equivalently, $\operatorname{Ker} f$ is a direct summand of $M$. It is clear that every semisimple module, every Baer module is a Rickart module. We continue to investigate properties of Rickart modules.

Let $M$ be an $R$-module. A right $R$-module $N$ is called $M$-principally projective [9], if for any $f \in S$, and any $N \stackrel{h}{\rightarrow} f(M)$ there exists a $N \stackrel{g}{\rightarrow} M$ such that the following diagram is commutative.

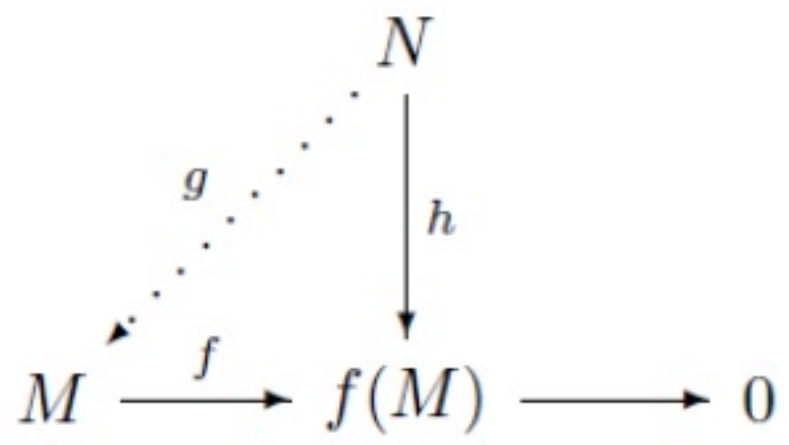

By the following Theorem 2.1 we investigate the relations between this class of modules and Rickart modules.

Theorem 2.1. Let $M$ be an R-module. Then $M$ is a Rickart module if and only if every right $R$-module is $M$-principally projective.

Proof. Assume that $M$ is a Rickart module and let $f \in S$. There exists $e^{2}=e \in S$ such that $r_{M}(f)=e M$. Then $M=r_{M}(f) \oplus K$ for some $K \leq M$. For any right $R$ module $N$ and any $N \stackrel{h}{\rightarrow} f(M)$, since $f(M) \cong M / r_{M}(f)$ for any $n \in N$ we may write $h(n)=k+r_{M}(f)$ for some $k \in K$ and we define $N \stackrel{g}{\rightarrow} M$ by $g(n)=k$. Then $g$ is a well defined $R$-map and for $n \in N, h(n)=f g(n)$. Conversely, suppose that every right $R$-module $N$ is $M$-principally projective and $f \in S$. In particular $M / r_{M}(f)$ is $M$-principally projective. So consider the identity map from $M / r_{M}(f)$ onto $M / r_{M}(f)$. By considering $f(M) \cong M / r_{M}(f)$ and supposition there exists a map $g$ from $M / r_{M}(f)$ to $M$ such that $1=f g$. For any $m \in M, m-g(f(m)) \in r_{M}(f)$ 
and $g(f(m)) \in I m g$, we have $M=r_{M}(f) \oplus I m g$. Let $e$ denote the projection of $M$ onto $r_{M}(f)$. Then $r_{M}(f)=e M$.

Let $M$ be an $R$-module and consider the set

$$
F(M)=\{m \in M \mid f m=0 \text { for some nonzero } f \in S\}
$$

of all torsion elements of the module $M$ with respect to $S$. The subset $F(M)$ of $M$ need not be a submodule of the modules ${ }_{S} M$ and $M_{R}$ in general. If $S$ is a commutative domain, then $F(M)$ is an $(S, R)$-submodule of $M$.

Proposition 2.2. Let $M$ be an $R$-module with a domain $S=\operatorname{End}_{R}(M)$. If $M$ is a Rickart module, then $F(M)=0$ and every nonzero element of $S$ is a monomorphism.

Proof. Let $M$ be a Rickart module and $0 \neq f \in S$. Then there exists an idempotent $e \in S$ such that $r_{M}(f)=e M$. Hence $f e M=0$. Thus $f e=0$ in $S$. Since $S$ is a domain and $f$ is nonzero, $e=0$ or every nonzero element of $S$ is a monomorphism. If $m \in F(M)$, then there exists a nonzero $f \in S$ such that $f m=0$. Since $f$ is a monomorphism, we have $m=0$, and so $F(M)=0$.

The following result is an immediate consequence of Proposition 2.2.

Corollary 2.3. Let $M$ be an $R$-module with a domain $S=\operatorname{End}_{R}(M)$. If $M$ is a Rickart module, then $M$ is torsion-free.

The next result can be obtained from Proposition 2.2 and [7, Theorem 2.23].

Corollary 2.4. Let $M$ be an R-module. Then the following are equivalent.

(1) $M$ is an indecomposable Baer module.

(2) $S$ is a domain and $M$ is a Rickart module.

(3) Every nonzero element of $S$ is a monomorphism.

Our next endeavor is to investigate relationships among reduced, rigid, symmetric, semicommutative, Armendariz modules and their endomorphism rings by using Rickart modules.

Definition 2.5. Let $M$ be an $R$-module. A module $M$ is called $S$-reduced if $f m=0$ implies $\operatorname{Im} f \cap S m=0$ for each $f \in S, m \in M$.

It can be easily proved that $M$ is an $S$-reduced module if and only if $f^{2} m=0$ implies $f S m=0$ for each $f \in S, m \in M$. 
Lemma 2.6. Let $M$ be an $R$-module. If $M$ is an $S$-reduced module, then $S$ is a reduced ring. The converse holds if $M$ is a Rickart module.

Proof. The first statement is clear from [1, Lemma 2.11] and [1, Proposition 2.14]. Conversely, assume that $M$ is a Rickart module and $S$ is a reduced ring. Let $f \in S$ and $m \in M$ with $f m=0$. Then $r_{M}(f)=e M$ for some $e^{2}=e \in S$. Hence $f e=0$ and $m=e m$. Since $e$ is central, we have $e f=0$. Let $f m_{1}=g m \in f M \cap S m$, where $m_{1} \in M$ and $g \in S$. Thus $0=$ ef $m_{1}=e g m=g e m=g m$, and so $f M \cap S m=0$. Therefore $M$ is $S$-reduced.

Let $M$ be an $R$-module. Recall that $M$ is called an $S$-rigid module [1] if for any $f \in S$ and $m \in M, f^{2} m=0$ implies $f m=0$.

Lemma 2.7. Let $M$ be an R-module. If $M$ is an $S$-rigid module, then $S$ is a reduced ring. The converse holds if $M$ is a Rickart module.

Proof. The first statement is clear from [1, Lemma 2.20]. Conversely, assume that $M$ is a Rickart module and $S$ is a reduced ring. Let $f \in S$ and $m \in M$ with $f^{2} m=0$. Then $r_{M}(f)=e M$ for some $e^{2}=e \in S$. Hence $f e=0$ and $f m=e f m$. Since $e$ is central, we have $f m=e f m=f e m=0$. Therefore $M$ is $S$-rigid.

According to Lambek [4], a ring $R$ is called symmetric if whenever $a, b, c \in R$ satisfy $a b c=0$, we have $a c b=0$. For the module case, we have the following.

Definition 2.8. Let $M$ be an $R$-module. A module $M$ is called $S$-symmetric if for any $m \in M$ and $f, g \in S, f g m=0$ implies $g f m=0$.

Lemma 2.9. Let $M$ be an R-module. If $M$ is an $S$-symmetric module, then $S$ is a symmetric ring. The converse holds if $M$ is a Rickart module.

Proof. Let $f, g, h \in S$ and assume $f g h=0$. Then $f g(h(m))=0$ and $g(f h)(m)=0$ implies $f h g(m)=0$ for all $m \in M$. Hence $f h g=0$. Conversely, assume that $M$ is a Rickart module and $S$ is a symmetric ring. Let $f, g \in S$ and $m \in M$ with $f g m=0$. Then $r_{M}(f g)=e M$ for some $e^{2}=e \in S$. Hence $f g e=0$ and $m=e m$. By assumption $g e f=0$. Since $e$ is central, we have $g f m=g f e m=g e f m=0$. Therefore $M$ is $S$-symmetric.

Lemma 2.10. Let $M$ be an $R$-module. If $M$ is an $S$-semicommutative module, then $S$ is a semicommutative ring. The converse holds if $M$ is Rickart. 
Proof. The first statement is proved in [2, Lemma 2.1]. Conversely, assume that $M$ is a Rickart module and $S$ is a semicommutative ring. Let $f \in S, m \in M$ with $f m=0$. Then $r_{M}(f)=e M$ for some $e^{2}=e \in S$. Hence $f e=0$ and $m=e m$. Since $e$ is central, $f g m=f g e m=f e g m=0$ for any $g \in S$. Thus $M$ is $S$-semicommutative.

In [6], the ring $R$ is called Armendariz if for any $f(x)=\sum_{i=0}^{n} a_{i} x^{i}$, $g(x)=\sum_{j=0}^{s} b_{j} x^{j} \in R[x], f(x) g(x)=0$ implies $a_{i} b_{j}=0$ for all $i$ and $j$. Let $M$ be an $R$-module. The module $M$ is called $S$-Armendariz if the following condition (1) is satisfied, while $M$ is said to be $S$-Armendariz of power series type if the following condition (2) is satisfied.

(1) For any $f(x)=\sum_{i=0}^{s} a_{i} x^{i} \in S[x]$ and $m(x)=\sum_{j=0}^{n} m_{j} x^{j} \in M[x], f(x) m(x)=0$ implies $a_{i} m_{j}=0$ for all $i$ and $j$.

(2) For any $f(x)=\sum_{i=0}^{\infty} a_{i} x^{i} \in S[[x]]$ and $m(x)=\sum_{j=0}^{\infty} m_{j} x^{j} \in M[[x]], f(x) m(x)=$ 0 implies $a_{i} m_{j}=0$ for all $i$ and $j$.

Lemma 2.11. Let $M$ be an $R$-module. If $M$ is an $S$-Armendariz module, then $S$ is an Armendariz ring. The converse holds if $M$ is a Rickart module.

Proof. Let $f(x)=\sum_{i=0}^{n} a_{i} x^{i}, g(x)=\sum_{j=0}^{k} b_{j} x^{j} \in S[x]$ with $f(x) g(x)=0$. For any $m \in$ $M, g(x) m=\sum_{j=0}^{k}\left(b_{j} m\right) x^{j} \in M[x]$. Since $f(x) g(x)=0$, we have $f(x)(g(x) m)=0$. This implies that $a_{i}\left(b_{j} m\right)=\left(a_{i} b_{j}\right) m=0$ for all $0 \leq i \leq n$ and $0 \leq j \leq k$, and so $a_{i} b_{j}=0$ for all $i$ and $j$. Therefore $S$ is Armendariz. Conversely, assume that $S$ is an Armendariz ring and $M$ is a Rickart module. By [5, Proposition 3.2], $S$ is a right Rickart ring. Since $S$ is Armendariz, $S$ is a reduced ring. By Lemma 2.6, $M$ is $S$-reduced and so $S$-Armendariz.

Corollary 2.12. Let $M$ be an R-module. If $M$ is an Armendariz of power series type, then $S$ is an $S$-Armendariz of power series type. The converse holds if $M$ is a Rickart module.

Proof. Similar to the proof of Lemma 2.11. 
We now summarize the relations between rigid, reduced, symmetric, semicommutative, Armendariz modules and their endomorphism rings by using Rickart modules.

Theorem 2.13. Let $M$ be an R-module. If $M$ is a Rickart module, then

(1) $M$ is $S$-rigid if and only if $S$ is a reduced ring.

(2) $M$ is $S$-reduced if and only if $S$ is a reduced ring.

(3) $M$ is $S$-symmetric if and only if $S$ is a symmetric ring.

(4) $M$ is $S$-semicommutative if and only if $S$ is a semicommutative ring.

(5) $M$ is $S$-Armendariz if and only if $S$ is an Armendariz ring.

(6) $M$ is $S$-Armendariz of power series type if and only if $S$ is an Armendariz of power series type ring.

Proof. (1) Lemma 2.6. (2) Lemma 2.7. (3) Lemma 2.9. (4) Lemma 2.10. (5)

Lemma 2.11. (6) Corollary 2.12.

The next result follows from Theorem 2.13 and [1, Theorem 2.25].

Corollary 2.14. Let $M$ be an R-module. If $M$ is a Rickart module, then the following conditions are equivalent.

(1) $S$ is a reduced ring.

(2) $S$ is a symmetric ring.

(3) $S$ is a semicommutative ring.

(4) $S$ is an Armendariz ring.

(5) $S$ is an Armendariz of power series type ring.

In the sequel, we study the polynomial extension of Rickart modules. Let $M$ be an $R$-module. It can be easily shown that $M[x]=\left\{\sum_{i=0}^{s} m_{i} x^{i}: s \geq 0, m_{i} \in M\right\}$ is an abelian group under an obvious addition operation and $M[x]$ becomes a module over $R[x]$ with

$$
\begin{gathered}
m(x)=\sum_{i=0}^{s} m_{i} x^{i} \in M[x] \quad, \quad f(x)=\sum_{i=0}^{t} a_{i} x^{i} \in R[x], \\
m(x) f(x)=\sum_{k=0}^{s+t}\left(\sum_{i+j=k} m_{i} a_{j}\right) x^{k} .
\end{gathered}
$$


Similarly, $M[x]$ is a left $S[x]$-module with

$$
\begin{gathered}
f(x)=\sum_{i=0}^{t} f_{i} x^{i} \in S[x] \quad, \quad m(x)=\sum_{j=0}^{s} m_{j} x^{j} \in M[x], \\
f(x) m(x)=\sum_{k=0}^{s+t}\left(\sum_{i+j=k} f_{i} m_{j}\right) x^{k} .
\end{gathered}
$$

The module $M[x]$ is called Rickart with respect to $S[x]$ if for any $f(x) \in S[x]$, there exists $e(x)^{2}=e(x) \in S[x]$ such that $r_{M[x]}(f(x))=e(x) M[x]$.

Theorem 2.15. Let $M$ be an $R$-module with $S=\operatorname{End}_{R}(M)$. If $M[x]$ is a Rickart module with respect to $S[x]$, then $M$ is Rickart. The converse holds if $M$ is $S$ Armendariz.

Proof. Assume that $M[x]$ is a Rickart module and $f \in S$. Consider $\bar{f} \in S[x]$ defined by $\bar{f}\left(\sum m_{i} x^{i}\right)=\sum f\left(m_{i}\right) x^{i}$. Then $\operatorname{Ker} \bar{f}$ is a direct summand of $M[x]$, that is, $M[x]=\operatorname{Ker} \bar{f} \oplus K$. It is easy to show that $M=\operatorname{Ker} f \oplus K_{0}$, where $K_{0}$ is the set of elements in $K$ evaluated in zero. Then $M$ is a Rickart module. Conversely, assume that $M$ is a Rickart module and $f(x)=\sum_{i=0}^{k} f_{i} x^{i} \in S[x]$. By hypothesis, there exist $e_{i}^{2}=e_{i} \in S(i=0,1,2, \ldots, k)$ such that $r_{M}\left(f_{i}\right)=e_{i} M$. Let $e=e_{0} e_{1} e_{2} \ldots e_{k}$. We prove $r_{M[x]}(f(x))=e M[x]$. For if $m(x)=\sum_{j=0}^{t} m_{j} x^{j} \in r_{M[x]}(f(x))$, then $f(x) m(x)=0$. Since $M$ is $S$-Armendariz, $f_{i} m_{j}=0$ for each $i=0,1,2, \ldots, k$ and for each $j=0,1,2, \ldots, t$. Then $m_{j} \in r_{M}\left(f_{i}\right)=e_{i} M$ and so $e_{i} m_{j}=m_{j}, e m_{j}=m_{j}$ and $e m(x)=m(x)$. Hence $m(x) \in e M[x]$ and so $r_{M[x]}(f(x)) \leq e M[x]$. On the other hand, $e M[x] \leq r_{M[x]}(f(x))$ and so $e M[x]=r_{M[x]}(f(x))$.

Then we have the following result.

Corollary 2.16. Let $R$ be a ring. If $R[x]$ is a left Rickart ring, then $R$ is a left Rickart ring. The converse holds if $R$ is Armendariz.

Özet: $R$ birimli bir halka, $M$ sağ $R$-modül ve $M$ nin endomorfizma halkası $S=$ $\operatorname{End}_{R}(M)$ olsun. Her $f \in S$ için $r_{M}(f)=e M$ olacak biçimde $e^{2}=e \in S$ varsa (denk olarak Ker $f, M$ modülünün bir direkt toplananı ise) $M$ ye Rickart modül adı verilmiştir [8]. Bu çalışmada Rickart modüllerin özellikleri incelenmeye devam edilmiştir. $M$ bir Rickart modül olmak üzere, $M$ nin $S$-katı (sirasıyla $S$-indirgenmiş, $S$-simetrik, $S$-yarı değişmeli, $S$-Armendariz) modül olması için gerek ve yeter şartın $S$ nin katı (sırasıyla indirgenmiş, simetrik, yarı değişmeli, Armendariz) halka olduğu gösterilmiştir. $M[x], S[x]$ halkasına 
göre Rickart modül iken $M$ nin de Rickart modül olduğu, tersinin $M$ nin $S$-Armendariz olması durumunda doğru olduğu ispatlanmıştır. Ayrıca bir $M$ modülünün Rickart olması için gerek ve yeter şartın her să̆ modülün $M$-temel projektif olduğu elde edilmiştir.

\section{REFERENCES}

[1] N. Agayev, S. Halicioglu and A. Harmanci, On Rickart modules, Bulletin of the Iranian Mathematical Society, 38(2) (2012), 433-445.

[2] N. Agayev, T. Ozen and A. Harmanci, On a Class of Semicommutative Modules, Proc. Indian Acad. Sci. 119(2009), 149-158.

[3] I. Kaplansky, Rings of Operators, Math. Lecture Note Series, Benjamin, New York, 1965.

[4] J. Lambek, On the representation of modules by sheaves of factor modules, Canad. Math. Bull. 14(1971), 359-368.

[5] G. Lee, S. T. Rizvi and C. S. Roman, Rickart Modules, Comm. Algebra 38(11)2010, 40054027.

[6] M. B. Rege and S. Chhawchharia, Armendariz Rings, Proc. Japan Acad. Ser. A Math. Sci. 73(1997), 14-17.

[7] S. T. Rizvi and C. S. Roman, Baer and Quasi-Baer Modules, Comm. Algebra 32(2004), 103-123.

[8] S. T. Rizvi and C. S. Roman, On direct sums of Baer modules, J. Algebra 321(2009), 682-696.

[9] H. Tansee and S. Wongwai, A note on semi-projective modules, Kyungpook Math. J. 42(2002), 369-380.

Current address:, Burcu Üngör, Sait Halıcığlu : Ankara University, Faculty of Sciences, Dept. of Mathematics, Ankara, TURKEY., Gizem Kafkas: Department of Mathematics, Izmir Institute of Technology, TURKEY., Abdullah Harmanci: Department of Mathematics, Hacettepe University, TURKEY.

E-mail address: bungor@science.ankara.edu.tr, gizemkafkas@iyte.edu.tr, halici@ankara.edu.tr, harmanci@hacettepe.edu.tr

$U R L:$ http://communications.science $\cdot$ ankara.edu.tr/index $\cdot$ php? series=A1 